\title{
A Study of Prevalence of Diabetes and Prediabetes in Patients of Non-Alcoholic Fatty Liver Disease and the Impact of Diabetes on Liver Histology in Coastal Eastern India
}

\author{
Shivaram Prasad Singh ${ }^{*}$, Ayaskanta Singh1, Girish Kumar Pati1, Bijay Misra1, \\ Debasis Misra1, Sanjib Kumar Kar', Manas Kumar Panigrahi', Chudamani Meher ${ }^{2}$, \\ Omprakash Agrawal'2, Niranjan Rout ${ }^{3}$, Kaumudi Pattnaik ${ }^{4}$, \\ Pallavi Bhuyan", Amarendra Mohapatra ${ }^{5}$ \\ ${ }^{1}$ Department of Gastroenterology, S. C. B. Medical College, Cuttack, India \\ ${ }^{2}$ Department of Radiology, Beam Diagnostics Centre, Cuttack, India \\ ${ }^{3}$ Department of Oncopathology, A. H. Regional Cancer Center, Cuttack, India \\ ${ }^{4}$ Department of Pathology, S. C. B. Medical College, Cuttack, India \\ ${ }^{5}$ Regional Medical Research Centre, Bhubaneswar, India \\ Email: scb gastro dept@hotmail.com
}

Received 20 August 2014; revised 15 September 2014; accepted 6 October 2014

Copyright (C) 2014 by authors and Scientific Research Publishing Inc.

This work is licensed under the Creative Commons Attribution International License (CC BY). http://creativecommons.org/licenses/by/4.0/

(c) (i) Open Access

\section{Abstract}

Background and Objectives: Non-alcoholic fatty liver disease (NAFLD) is more common in diabetic patients. There are limited studies on clinical, biochemical and histological features of NAFLD patients who are diabetic. The aim of the study was to determine the prevalence of diabetes and prediabetes in a cohort of NAFLD patients and to compare anthropometry, biochemical and metabolic parameters and hepatic histology of diabetic NAFLD patients with non-diabetic NAFLD patients. Methods: 515 consecutive NAFLD patients diagnosed by abdominal ultrasound and 100 healthy controls were subjected to detailed anthropometric measurements and biochemical assays including blood sugars, LFT, lipid profile and HOMA. Diabetes and prediabetes were defined according to WHO criteria. Patients were categorized and compared according to the presence or absence of diabetes. Liver biopsy was performed in 240 NAFLD patients and the liver histology was also compared between the two groups. Statistical analysis was performed on SPSS 16. Results: 124 out of the $515(24.08 \%)$ NAFLD patients were diabetics, 118 out of $515(22.9 \%)$ were pre-dia-

${ }^{*}$ Corresponding author. 
betics, while only 3 out of 100 controls had impaired glucose tolerance. Diabetic patients were older. NAFLD patients with diabetes had significantly higher waist circumference $[98.02 \pm 12.01 \mathrm{vs}$ $93.89 \pm 8.8, p=0.000]$ as compared to the NAFLD patients without diabetes. Fasting blood sugar $[124 \pm 46.3$ vs $90.8 \pm 10.2, p=0.000]$, triglyceride level $[218.4 \pm 17.6$ vs $192 \pm 9, p=0.03]$ and HOMA-IR $[2.6 \pm 0.36$ vs $1.84 \pm 0.2, p<0.001]$ were significantly higher in NAFLD-diabetes group. Hypertension [ $35 \%$ vs $11.7 \%, p=0.000]$ was commoner in diabetic NAFLD patients. Histopathology in the diabetic patients revealed steatosis alone in $34.2 \%$ cases, borderline NASH in $31.4 \%$ and definite NASH in $34.2 \%$. Fatty change alone was noted in $16.5 \%$ cases, borderline NASH in $34.1 \%$, while $49 \%$ had definite NASH on liver biopsy of NAFLD patients without diabetes. Fibrosis was noted in $31.4 \%$ in diabetic and $27 \%$ in non-diabetic patients. IR alone had a linear correlation with necroinflammatory activity. Conclusion: The prevalence of diabetes and prediabetes is six times more in NAFLD patients than in healthy controls. NAFLD patients with diabetes have higher metabolic risk factors such as large waistline, hypertension, high triglyceride levels and increased insulin resistance. Diabetes or pre diabetes patients per se do not have histologically severe disease, rather insulin resistance play an important role in increasing the severity of the disease.

\section{Keywords}

Fatty Liver, Diabetes, Prediabetes, Liver Histology, Insulin Resistance, Steatohepatitis

\section{Introduction}

Non-alcoholic fatty liver disease (NAFLD) is a spectrum which encompasses simple steatosis (simple fatty liver), non alcoholic steatohepatitis (NASH), cirrhosis and even hepatocellular carcinoma (HCC) [1] [2]. NAFLD is highly prevalent in Western countries [1]. Studies from different regions of India [3]-[5] have shown that the prevalence of NAFLD has increased over the past two decades to become the commonest liver disease in Indians.

The presence of hyperinsulinemia or insulin resistance (IR) and their association with some of the features of the metabolic syndrome suggest that NAFLD might be the liver component of the metabolic syndrome [6]. Indians have a high prevalence of insulin resistance and the metabolic syndrome. Currently, non-alcoholic fatty liver disease (NAFLD) is considered to be an integral part of the metabolic syndrome with insulin resistance as a central pathogenic factor.

The profiles of NAFLD patients in India appear to be different from the Western population as they have a lower frequency of metabolic syndrome, lesser degree of adiposity, and histological milder disease at presentation [7] [8]. Increasing evidence from Western countries suggests that patients with diabetes mellitus (DM) are particularly at risk for developing the progressive form of NAFLD, i.e., NASH [9] [10]. However, a study by Das et al. from India showed positive correlation of insulin resistance with higher grades of NAFLD, rather than the degree of hyperglycemia in patients with type 2 DM [11]. A head on comparative study between diabetic NAFLD patients with non-diabetic NAFLD patient with special emphasis on liver histology has not been done in Indian population.

Whether diabetic NAFLD patients have a more severe disease, higher necroinflammatory activity and more prone to develop fibrosis as compared to non-diabetic NAFLD patients need to be evaluated.

The aim of the study was to document the prevalence of type 2 diabetes mellitus and pre-diabetes in patients with NAFLD and to determine their anthropometric, biochemical and metabolic profile. The other aim is to determine whether these diabetic NAFLD patients have more severe disease on their liver histology than those NAFLD patients with normal glycemic status.

\section{Materials and Methods}

This was a single centre observational analytical study of subjects attending a gastroenterology clinic at Cuttack, Orissa in coastal Eastern India from October 2012-October 2013. The study was approved by the KGF Ethical Committee. 
515 consecutive NAFLD patients and 100 controls were analysed in this study.

Patients: These were NAFLD patients $(n=515)$. The diagnosis of NAFLD was made on the basis of ultrasonography and histological confirmation whenever possible. Subjects were considered as cases if they presented with fatty liver defined according to the standard criteria accepted by the American Gastroenterology Association: An increase in hepatic echogenicity taking renal echogenicity as a reference, the presence of enhancement and lack of differentiation in periportal intensity and the vascular wall due to great hyperechogenicity of the parenchyma.

Controls: These were patients who had a normal ultrasonography which was performed for various causes such as pain abdomen, dyspepsia etc.

Exclusion criteria: Patients and controls consuming alcohol $>20 \mathrm{~g}$ /day, having other known liver diseases (hepatitis viruses A to E, autoimmune disease, Wilson's disease, alpha 1 anti-trypsin deficiency and haemochromatosis) and those on medications known to induce fatty liver or insulin sensitization such as estrogens, amiodarone, methotrexate, tamoxifen, glitazones and metformin were excluded.

The anthropometric assessment included measurements of weight, stature, and waist circumference (WC) and hip circumference (HC). Body mass index (BMI) was calculated as weight $(\mathrm{kg}) / \mathrm{stature}\left(\mathrm{m}^{2}\right)$. The WC and HC were measured at the level midway between the lowest rib and the iliac crest and at the level of the great trochanter.

Measurements of fasting glucose, triglycerides, cholesterol, and high-density lipoprotein (HDL) cholesterol, liver function tests were performed by standard laboratory methods. Insulin was measured by radioimmunoassay.

Diabetes mellitus and prediabetes was diagnosed by WHO criteria. NAFLD patients with diabetes were taken as one group and these patients were compared with the non-diabetic NAFLD group. IR was calculated using the homeostatic model assessment (HOMA) method. HOMA-IR uses a mathematical model derived from fasting blood glucose and plasma insulin [12]. Although a HOMA score of less than 1.0 is the ideal, the study by Bonora et al., found a mean HOMA-IR score of $2.06 \pm 0.14$ in the normal non-diabetic population [13]. Study done in our population had shown that the normal value of insulin resistance as assessed by HOMA-IR is less than 2 . For the purpose of calculation HOMA-IR value above 2 was considered insulin resistance in our study [14].

Liver biopsy was done in 240 patients who gave consent for biopsy. Liver biopsy was done using Bard Biopsy Gun 16 G, through intercoastal approach. Grading and staging of liver histopathology was done as per the classification proposed by Kleiner $e t$ al. using necroinflammatory activity for grading of NASH [15].

Statistics: Normally distributed continuous variables were expressed as mean \pm SD, and the continuous variables with skewed distribution were expressed as median (range). Student's t-test for unpaired data was used to compare groups when variables were normally distributed; otherwise, the Mann-Whitney test was used. Chi square test was used to compare differences in categorical variables. All analysis were done in SPSS software version 17 (SPSS Inc., Chicago, IL). A p value of less than 0.05 was considered significant.

\section{Results}

515 consecutive NAFLD patients were enrolled in the study. 100 healthy controls were also evaluated.

124 (24.08\%) NAFLD patients were diabetics and 118 (22.9\%) were pre-diabetics while only 3 out of 100 controls had diabetes. When the cohort of Type 2 DM with NAFLD was compared with non-diabetes NAFLD patients (Table 1), it was seen that diabetic NAFLD patients were older (mean age $46.6 \pm 10$ versus $40.47 \pm 9.8$, $\mathrm{p}$ value $=0.000$ ). The gender ratio, height, weight and BMI of NAFLD patients with diabetes were comparable with the non diabetes group. But central obesity was more common in those NAFLD patients who had Type 2 $\mathrm{DM}$ as they had significantly higher waist circumference [98.02 $\pm 12.01 \mathrm{vs} 93.89 \pm 8.8, \mathrm{p}=0.000]$ as compared to the NAFLD patients without diabetes. Another measurement of visceral obesity, i.e. waist height ratio was also higher in the former group. Metabolic parameters like fasting blood sugar [124 \pm 46.3 vs $90.8 \pm 10.2$, p = 0.000], triglyceride level [218.4 \pm 17.6 vs $192 \pm 9$, p $=0.03$ ] were significantly higher in NAFLD-Diabetes group. Paradoxically higher HDL [ $49.4 \pm 4.8$ vs $42.4 \pm 2.3$, p $=0.000$ ] was seen the non-diabetic group. Insulin resistance as measured by HOMA-IR was naturally higher in NAFLD group with diabetes [2.6 \pm 0.36 vs $1.84 \pm$ 0.2 , p $<0.001$ ]. Hypertension [ $48.4 \%$ vs $11.7 \%$, OR $=7.3$ ] was commoner in diabetic NAFLD patients. Thus most of the components of metabolic syndrome clustered in the NAFLD-diabetes group as compared to non diabetic NAFLD group. 
Table 1. Baseline parameters comparison between Diabetic NAFLD patients and Non-Diabetic NAFLD patients.

\begin{tabular}{|c|c|c|c|}
\hline Parameters & NAFLD with Type 2 DM & NAFLD without Type 2 DM & p Value* \\
\hline AGE & $46.6 \pm 10.4$ & $40.47 \pm 9.8$ & 0.000 \\
\hline Males & $93 / 124$ & $317 / 391$ & NS \\
\hline Height & $162.8 \pm 8.2$ & $163.4 \pm 8$ & NS \\
\hline Weight & $70.4 \pm 11.7$ & $70.4 \pm 11.3$ & NS \\
\hline BMI & $26.6 \pm 3.9$ & $26.3 \pm 3.9$ & NS \\
\hline Waist & $98.04 \pm 12$ & $93.89 \pm 8.8$ & 0.000 \\
\hline Hip & $100.8 \pm 10$ & $97.9 \pm 7.6$ & NS \\
\hline Waist/Hip Ratio & $0.98 \pm 0.1$ & $0.95 \pm 0.06$ & NS \\
\hline Waist/Height Ratio & $0.6 \pm 0.08$ & $0.57 \pm 0.07$ & 0.000 \\
\hline Hypertension & $60 / 124$ & $44 / 391$ & 0.000 \\
\hline FBS & $124 \pm 46.3$ & $90.8 \pm 10.2$ & 0.000 \\
\hline PPBS & $201 \pm 8.7$ & $122 \pm 5.1$ & 0.000 \\
\hline Triglyceride & $218.4 \pm 17.6$ & $192 \pm 9$ & 0.03 \\
\hline HDL & $49.4 \pm 4.8$ & $42.4 \pm 2.3$ & 0.000 \\
\hline Cholesterol & $183.8 \pm 65$ & $186.5 \pm 49$ & NS \\
\hline LDL & $103 \pm 41.2$ & $109 \pm 37.6$ & NS \\
\hline AST & $41.04 \pm 25.8$ & $42.44 \pm 22.8$ & NS \\
\hline ALT & $50.87 \pm 29.3$ & $51.5 \pm 39.4$ & NS \\
\hline HOMA IR & $2.6 \pm 0.36$ & $1.84 \pm 0.2$ & 0.000 \\
\hline
\end{tabular}

TG: Triglyceride; CHOL: Cholesterol; BMI: Body Mass Index; HDL: High Densitylipoprotein; LDL: Low Density Lipoprotein; FBG: Fasting Blood Glucose; PGBG: Postglucose Blood Glucose; AST: Aspartate Transaminase; ALT: Alanine Transaminases; HOMA IR: Homeostatic Model Assessment of Insulin Resistance; NS: Non Significant. "Significant $\mathrm{p}<0.05$.

Liver biopsy was done in 240 NAFLD patients, out of which 70 were diabetics. The hepatic histopathological comparison between the two groups is shown in Table 2. Surprisingly the necroinflammatory activity as measured by lobular inflammation was more in the non diabetes group as compared to the diabetic group. Hepatic steatosis, ballooning and NAFLD Activity Score (NAS) were comparable in both the groups. Most of the patients had mild fibrosis and both the groups were comparable with regards to presence of fibrosis in liver histology.

Histopathology in the diabetic patients revealed only fatty change in 24 (34.2\%) cases, borderline NASH in 22 (31.4\%) cases and definite NASH in 24 (34.2\%) as shown in Table 2. Fatty change alone was noted in 16.5\% cases, borderline NASH in $34.1 \%$, while $49 \%$ had definite NASH on liver biopsy of NAFLD patients without diabetes. Fibrosis was noted in 22/70 (31.4\%) in diabetic and 47/170 (27\%) in non-diabetic patients.

The diabetes and prediabetes group were grouped together and their histology was compared with those NAFLD patients with normal glycemic status (Table 3). Again the results were similar with higher lobular inflammation in the group with normal glycemic status and the rest other features of liver histology was comparable in both the groups.

The NAFLD cohort was divided into two groups according to the presence or absence of Insulin Resistance (IR) and the histological features were compared (Table 4). IR was present in around $60 \%$ of patients who had a liver biopsy. These patients had higher steatosis and more necroinflammatory activity and higher NAS as compared to those patients without IR. However, IR did not impact development of fibrosis. 
Table 2. Liver histology of NAFLD and relationship with diabetes mellitus.

\begin{tabular}{cccc}
\hline Histology & Diabetes NAFLD $(\mathrm{N}=70)$ & Non-Diabetes NAFLD (N = 170) & p Value $^{*}$ \\
\hline Moderate steatosis & $28(40 \%)$ & $72(42.4 \%)$ & 0.145 \\
Severe steatosis & $10(14.2 \%)$ & $30(17.6 \%)$ & 0.571 \\
Lobular inflammation & $42(60 \%)$ & $145(85 \%)$ & 0.001 \\
Ballooning & $47(67.1 \%)$ & $128(75.3 \%)$ & 0.204 \\
Fibrosis & $22(31.4 \%)$ & $47(27.6 \%)$ & 0.63 \\
Not NASH (only steatosis) & $24(34.2 \%)$ & $28(16.5 \%)$ & 0.003 \\
Borderline NASH & $22(31.4 \%)$ & $58(34.11 \%)$ & 1.0 \\
NASH & $24(34.4 \%)$ & $84(49.4 \%)$ & 0.03 \\
NAS & $3.5 \pm 1.7$ & $4.1 \pm 1.5$ & 0.06 \\
\hline
\end{tabular}

NASH: Non-Alcoholic Steatohepatitis; NAS: NAFLD Activity Score; "Significant p $<0.05$.

Table 3. Comparison of histology between diabetic and prediabetic NAFLD patients with those with normal glycemic status.

\begin{tabular}{cccc}
\hline Histology & NAFLD with Diabetes + Prediabetes $(\mathrm{N}=107)$ & NAFLD without Diabetes or IGT $(\mathrm{N}=133)$ & $\mathrm{p}$ Value \\
\hline Moderate steatosis & $45(42 \%)$ & $55(41.3 \%)$ & 1.0 \\
Severe steatosis & $17(15.9 \%)$ & $23(17.3 \%)$ & 0.86 \\
Lobular inflammation & $72(67.3 \%)$ & $115(86.5 \%)$ & 0.005 \\
Ballooning & $78(72.9 \%)$ & $98(73.7 \%)$ & 1.0 \\
Fibrosis & $32(29.9 \%)$ & $39(29.3 \%)$ & 1.0 \\
NAS & $3.7 \pm 1.7$ & $4.1 \pm 1.5$ & 0.06 \\
\hline
\end{tabular}

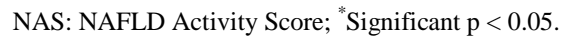

Table 4. Comparison of liver histology between the two NAFLD groups with and without IR.

\begin{tabular}{|c|c|c|c|}
\hline Histology & NAFLD with IR $(\mathrm{N}=72)$ & NAFLD without IR $(\mathrm{N}=50)$ & p Value ${ }^{*}$ \\
\hline Moderate steatosis & $24(33.3 \%)$ & 27 (54\%) & 0.02 \\
\hline Severe steatosis & $25(34.7 \%)$ & $3(6 \%)$ & 0.000 \\
\hline Lobular inflammation & $62(86.11 \%)$ & $39(78 \%)$ & 0.32 \\
\hline Ballooning & $58(80.6 \%)$ & $30(60 \%)$ & 0.014 \\
\hline Fibrosis & $31(43 \%)$ & $16(32 \%)$ & 0.25 \\
\hline NAS & $4.45 \pm 1.6$ & $3.6 \pm 1.5$ & 0.009 \\
\hline
\end{tabular}

NAS: NAFLD Activity Score; IR: Insulin Resistance; "Significant $\mathrm{p}<0.05$.

\section{Discussion}

This study included a large cohort of 515 NAFLD patients and histopathological study was done in about half of these patients. The prevalence of diabetes was $24 \%$ and prevalence of prediabetes was around $23 \%$.This is quite high as compared to a previous Indian study from Chandigarh where the prevalence has been reported to be around $12 \%$ and 14\% respectively [8]. Diabetes was defined according to WHO criteria and the diabetic group included those patients who were known diabetic or had abnormal glycemic status.

When diabetic NAFLD patients were compared with non-diabetic NAFLD patients, the components of metabolic syndrome such central obesity, high triglyceride levels and hypertension were more common in the former 
group. This finding is not surprising considering that diabetes mellitus is a manifestation of metabolic syndrome, strongly associated with NAFLD. This establishes the fact that there is a strong association between the various components of metabolic syndrome, but it is difficult to ascertain whether diabetes is the risk factor for development of hepatic steatosis and other features of metabolic syndrome from this study.

Another interesting aspect of the study is regarding the role of diabetes in progression or severity of NAFLD. The histology of diabetic and pre diabetic patients is almost similar to the non-diabetic patients. Neither are they associated with higher necro-inflammatory activity nor are they associated with advanced fibrosis. This is in contrast from the studies from western countries where it is seen that diabetes is a positive risk factor in progression from steatosis and steatohepatitis [10] [16]. However, a study by Das et al from India has demonstrated that degree of hyperglycemia did not much have correlation with higher grades of NAFLD [11]. However, this study had limitation of a small sample size of around 40 patients. The most suitable explanation for this is that Indian patients have milder disease with minimal fibrosis and hyperglycemia may play a role in those patients with advanced steatohepatitis and fibrosis.

However, when the NAFLD patients were segregated into two groups according to insulin resistance, significant association with higher grades of steatohepatitis is seen in those patients who have insulin resistance. This is in agreement with previous studies which suggest that insulin resistance plays a key role in progression of the disease [11] [17]. However, other mechanisms must be working along with insulin resistance as only 2/3rd of the patients had insulin resistance and development of fibrosis did not correlate with IR.

The study is not without limitations. The duration of diabetes and its impact on NAFLD was not evaluated. This requires long-term prospective follow up studies which can also identify the chronological relationship of diabetes with NASH. Besides, the effect of treatment of diabetes was not studied.

In conclusion, the prevalence of diabetes and pre diabetes is high in NAFLD patients. The NAFLD patients with DM are associated with dyslipidemia, central obesity, hypertension and insulin resistance. Diabetes or prediabetes patients do not have histologically severe disease, rather insulin resistance play an important role in increasing the severity and progression of the disease.

\section{References}

[1] Farrell, G.C. and Larter, C.Z. (2006) Nonalcoholic Fatty Liver Disease: From Steatosis to Cirrhosis. Hepatology, 43, S99-S112. http://dx.doi.org/10.1002/hep.20973

[2] Bugianesi, E., Leone, N. and Vanni, E. (2002) Expanding the Natural History of Nonalcoholic Steatohepatitis: From Cryptogenic Cirrhosis to Hepatocellular Carcinoma. Gastroenterology, 123, 134-140. http://dx.doi.org/10.1053/gast.2002.34168

[3] Singh, S.P., Nayak, S., Swain, M., Rout, N., Mallick, R.N., Agarwal, O., Meher, C. and Rao, M. (2004) Prevalence of Nonalcoholic Fatty Liver Disease in Coastal Eastern India: A Preliminary Ultrasonographic Survey. Tropical Gastroenterology, 25, 76-79.

[4] Mohan, V., Farooq, S., Deepa, M., et al. (2009) Prevalence of Nonalcoholic Fatty Liver Disease in Urban South Indians in Relations to Different Grades of Glucose Intolerance and Metabolic Syndrome. Diabetes Research and Clinical Practice, 84, 84-91. http://dx.doi.org/10.1016/j.diabres.2008.11.039

[5] Amarapurkar, D., Kamani, P., Patel, R., Gupte, P., Kumar, P., Agal, S., et al. (2007) Prevalence of Non-Alcoholic Fatty Liver Disease: Population Based Study. Annals of Hepatology, 6, 161-163.

[6] Marchesini, G., Brizi, M., Bianchi, G., Tomassetti, S., Bugianesi, E., Lenzi, M., et al. (2001) Nonalcoholic Fatty Liver Disease, a Feature of the Metabolic Syndrome. Diabetes, 50, 1844-1850. http://dx.doi.org/10.2337/diabetes.50.8.1844

[7] Madan, K., Batra, Y., Gupta, S.D., Chander, B., Rajan, K.D., Tewatia, M.S., Panda, S.K. and Acharya, S.K. (2006) Nonalcoholic Fatty Liver Disease May Not Be a Severe Disease at Presentation among Asian Indians. World Journal of Gastroenterology, 12, 3400-3405.

[8] Duseje, A., Das, A., Das, R., Dhiman, R.K., Chawla, A., Bhansali, A., et al. (2007) The Clinicopathological Profile of Indian Patients with Nonalcoholic Fatty Liver Disease (NAFLD) Is Different from That in the West. Digestive Diseases and Sciences, 52, 2368-2374. http://dx.doi.org/10.1007/s10620-006-9136-y

[9] Angulo, P., Keach, J.C., Batts, K.P. and Lindor, K.D. (1999) Independent Predictors of Liver Fibrosis in Patients with Non-Alcoholic Steatohepatitis. Hepatology, 306, 1356-1362. http://dx.doi.org/10.1002/hep.510300604

[10] Dixon, J.B., Bhathal, P.S. and O’Brien, P.E. (2001) Nonalcoholic Fatty Liver Disease: Predictors of Nonalcoholic Steatohepatitis and Liver Fibrosis in the Severely Obese. Gastroenterology, 121, 91-100.

http://dx.doi.org/10.1053/gast.2001.25540 
[11] Das, S., Singh, S.P., Parida, P.K. and Mallick, R.N. (2010) Non-Alcoholic Fatty Liver Disease in Subjects with Type 2 Diabetes Mellitus and Non-Diabetics with Special Reference to Insulin Resistance and Hepatic Histopathological Changes. Diabetes \& Metabolic Syndrome: Clinical Research \& Reviews, 4, 226-229. http://dx.doi.org/10.1016/j.dsx.2010.07.004

[12] Matthews, D.R., Hosker, J.P., Rudenski, A.S., Naylor, B.A., Treacher, D.F. and Turner, R.C. (1985) Homeostasis Model Assessment: Insulin Resistance and Beta Cell Function from Fasting Plasma Glucose and Insulin Concentrations in Man. Diabetologia, 28, 412-419. http://dx.doi.org/10.1007/BF00280883

[13] Bonora, E., Targher, G., Alberiche, M., Bonadonna, R.C., Saggiani, F., Zenere, M.B., et al. (2000) Homeostasis Model Assessment Closely Mirrors the Glucose Clamp Technique in the Assessment of Insulin Sensitivity. Diabetes Care, 23, 57-63. http://dx.doi.org/10.2337/diacare.23.1.57

[14] Das, S., Bhoi, S.K., Baliarsinha, A.K. and Baig, M.A.A. (2007) Auto Immunity, Insulin Resistance and Beta Cell Function in Subjects with Low Body Weight, Type 2 Diabetes Mellitus. Metabolic Syndrome and Related Disorders, 5, 136-141. http://dx.doi.org/10.1089/met.2006.0024

[15] Kleiner, D.E., Brunt, E.M., Van Natta, M., Behling, C., Contos, J.M., Cummings, O., et al. (2005) Design and Validation of a Histological Scoring System for Nonalcoholic Fatty Liver Disease. Hepatology, 41, 1313-1321. http://dx.doi.org/10.1002/hep.20701

[16] Wanless, I.R. and Lentz, J.S. (1990) Fatty Liver Hepatitis (Steatohepatitis) and Obesity: An Autopsy Study with Analysis of Risk Factors. Hepatology, 12, 1106-1110. http://dx.doi.org/10.1002/hep.1840120505

[17] Amarapurkar, D. and Das, H.S. (2002) Chronic Liver Disease in Diabetes Mellitus. Tropical Gastroenterology, 23, 3-5. 
Scientific Research Publishing (SCIRP) is one of the largest Open Access journal publishers. It is currently publishing more than 200 open access, online, peer-reviewed journals covering a wide range of academic disciplines. SCIRP serves the worldwide academic communities and contributes to the progress and application of science with its publication.

Other selected journals from SCIRP are listed as below. Submit your manuscript to us via either submit@scirp.org or Online Submission Portal.
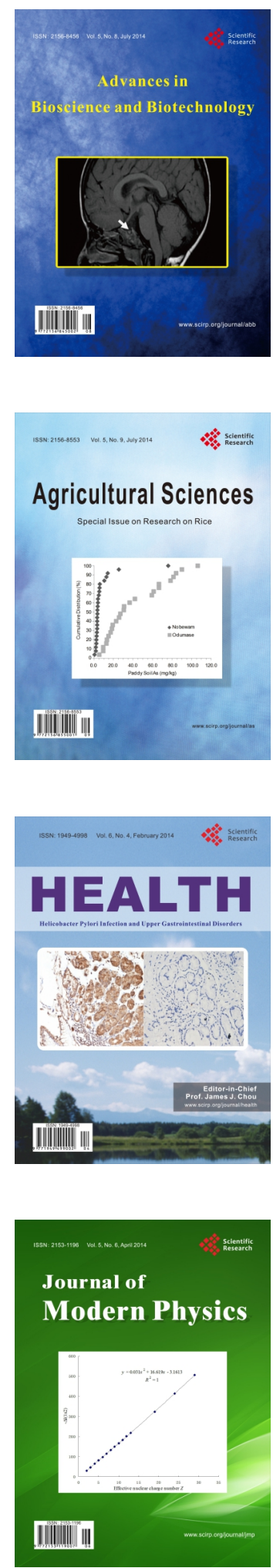
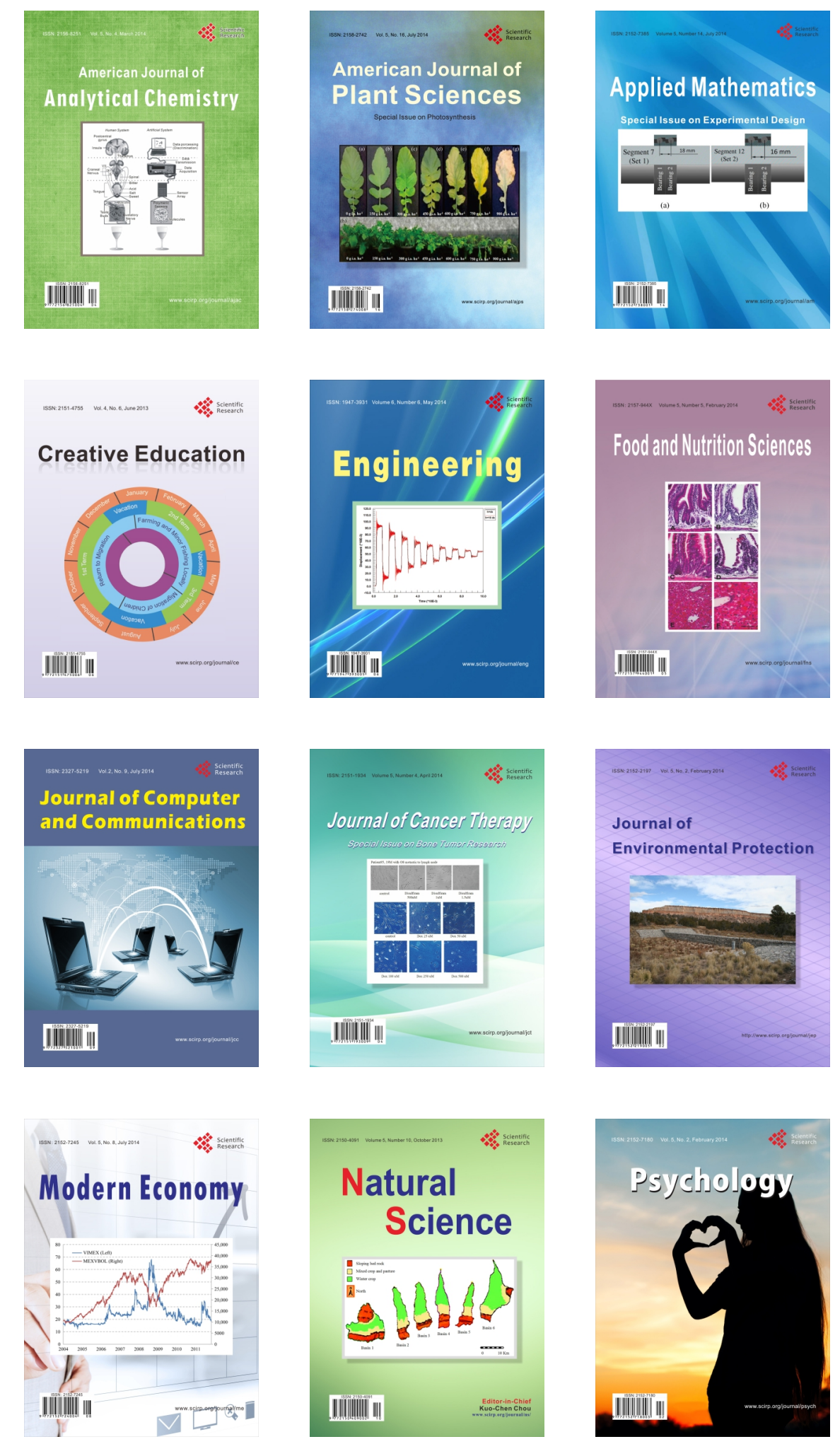